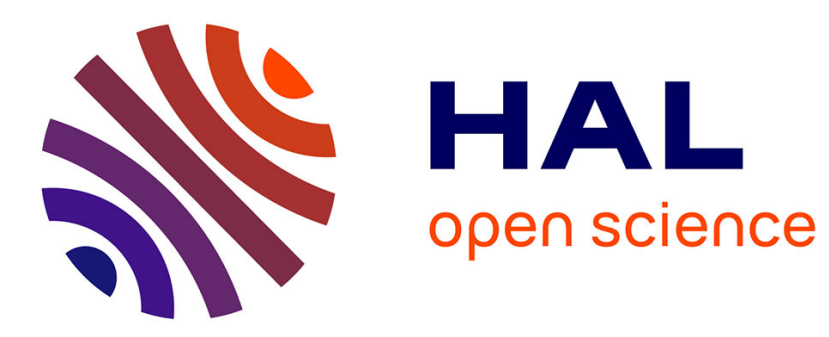

\title{
A model of influence with an ordered set of possible actions
}

\author{
Agnieszka Rusinowska, Michel Grabisch
}

\section{To cite this version:}

Agnieszka Rusinowska, Michel Grabisch. A model of influence with an ordered set of possible actions. Theory and Decision, 2010, 69 (4), pp.635-656. 10.1007/s11238-009-9150-6 . hal-00519413

\section{HAL Id: hal-00519413 \\ https://hal.science/hal-00519413}

Submitted on 20 Sep 2010

HAL is a multi-disciplinary open access archive for the deposit and dissemination of scientific research documents, whether they are published or not. The documents may come from teaching and research institutions in France or abroad, or from public or private research centers.
L'archive ouverte pluridisciplinaire HAL, est destinée au dépôt et à la diffusion de documents scientifiques de niveau recherche, publiés ou non, émanant des établissements d'enseignement et de recherche français ou étrangers, des laboratoires publics ou privés. 


\title{
A model of influence with an ordered set of possible actions
}

\author{
MICHEL GRABISCH${ }^{1}$ and AGNIESZKA RUSINOWSKA ${ }^{2}$ \\ 1 Université Paris I Panthéon-Sorbonne \\ Centre d'Economie de la Sorbonne, 106-112 Bd de l'Hôpital, 75013 Paris, France \\ Phone: 0033144078 285, Fax: 0033144078301 \\ michel.grabisch@univ-paris1.fr \\ 2 GATE, CNRS UMR 5824 - Université Lumière Lyon 2 \\ 93, Chemin des Mouilles - B.P.167, 69131 Ecully Cedex, France \\ Phone: 0033472866 080, Fax: 0033472866090 \\ rusinowska@gate.cnrs.fr
}

\begin{abstract}
In the paper, a yes-no model of influence is generalized to a multi-choice framework. We introduce and study weighted influence indices of a coalition on a player in a social network, where players have an ordered set of possible actions. Each player has an inclination to choose one of the actions. Due to mutual influence among players, the final decision of each player may be different from his original inclination. In a particular case, the decision of the player is closer to the inclination of the influencing coalition than his inclination was, i.e., the distance between the inclinations of the player and of the coalition is greater than the distance between the decision of the player and the inclination of the coalition in question. The weighted influence index which captures such a case is called the weighted positive influence index. We also consider the weighted negative influence index, where the final decision of the player goes farther away from the inclination of the coalition. We consider several influence functions defined in the generalized model of influence and study their properties. The concept of a follower of a given coalition, and its particular case, a perfect follower, are defined. The properties of the set of followers are analyzed.
\end{abstract}

\section{JEL Classification: C7, D7}

Keywords: weighted positive influence index, weighted negative influence index, influence function, follower of a coalition, perfect follower, kernel

Corresponding author: Agnieszka Rusinowska

\section{Introduction}

The point of departure of this research is the framework of influence introduced in [21], and later studied in detail, in particular, in [8,9,18-20,26-29]. In the original framework, a social network with players, also referred to as agents or actors, who have to make an acceptance-rejection decision, is considered. The original yes-no model assumes that each player has an inclination to say either 'yes' or 'no'. By the inclination of a player we mean a decision the player would make if he were to decide completely on his own. Then, each player makes his decision which, due to influence of other players, may be different from the original inclination of the player. Such a transformation of the inclinations into the decisions is represented by an influence function. The influence we are talking about can cover many aspects. One of the simplest examples of the influence is to follow our bosses, directors, parents, because deciding differently from their opinions could have bad consequences on our future position. The influence may also mean to follow our 
great authorities whose opinions we rely on. Also learning, observing the consequences of the others' decisions can be treated as a kind of influence.

As pointed out in [20], although the influence aspects incorporated into the social network make the framework especially attractive, the concepts introduced in the original model are not sufficient to study this framework. The main concept introduced in the original model [21] is the concept of decisional power (the Hoede-Bakker index) which, in fact, does not show the actual role of the influence function. In [20] we define and investigate the so called weighted influence indices that do measure influence between players in the yes-no model. Several special cases of the weighted influence indices are considered, like the possibility influence index, and the certainty influence index. Two kinds of influence, a direct influence and an opposite influence, are studied in [20]. Under the direct influence, an influencing coalition succeeds in making a player vote according to the inclination of the coalition, while the inclination of the player was different from the inclination of the coalition. The opposite influence of a coalition on a player means that the inclinations of the player and the coalition coincide, but the player's vote is different from this inclination. In [19] the model of influence in a social network is compared with the framework of command games introduced in $[23,24]$ (see also [30]). We show that our framework of influence is more general than the framework of the command games, i.e., in particular, we define several influence functions that capture the command structure.

Considering more than just two options 'yes' or 'no' in voting situations has been already incorporated in several works. For instance, voting systems with several levels of approval in the input and output, where those levels are qualitatively ordered, have been considered in [17]. The authors introduce $(j, k)$ simple games, in which each voter expresses one of $j$ possible levels of input support, and the output consists of one of $k$ possible levels of collective support. Standard simple games are $(2,2)$ simple games, and $(3,2)$ simple games allow each voter to have a middle option, which may be interpreted as abstention. In [16] an a priori Shapley-Shubik power index for such $(j, k)$ simple games is provided. In [15] the author considers a Banzhaf score and three Banzhaf measures in this framework. In particular, he provides an axiomatic characterization of the Banzhaf score for $(j, k)$ simple games, following a procedure closely related to the axiomatization for simple games given in [10]. Important works on abstention can also be found, in particular, in [7], and also in [14], where a considered game can be viewed as a special case of $(3,3)$ simple games. Also in [11-13], the authors study voting systems with abstention, and their ternary voting rules correspond to $(3,2)$ simple games with three input alternatives: 'yes', 'abstention', and 'no'. In [3-6] the author considers games with $n$ players and $r$ alternatives, where these alternatives are not ordered: each input alternative has its own coalition of supporting players, and each such coalition is assigned an output cardinal value. In [1] the values analyzed in [4] are extended for a coalition structure. In [2] the authors define closely related $r$-games and the Banzhaf-Coleman index for these games. In [22] multi-choice games and the Shapley value for these games are studied: the inputs are also ordered and the output is a single cardinal value.

The model of influence, originally introduced in [21] and later studied by other authors, is not the only framework of influence considered in the literature. There are other approaches to influence that are different from the approach adopted in [18-20]. For instance, the notion of influence relation in simple games has been introduced in [25] to qualitatively compare the a priori influence of voters in a simple game, where players can 
vote either 'yes' or 'no'. As defined in [25], in a simple game voter $i$ is said to be at least as influential as voter $j$, if whenever $j$ can transform a loosing coalition into a majority by joining it, voter $i$ can achieve the same ceteris paribus. In [31] the influence relation is extended to voting games with abstention, where abstention is an intermediate option between 'yes' and 'no'. A voting game with abstention consists of a non-empty set $\mathcal{W}$ of tripartitions of a set of voters, and $\left(S_{1}, S_{2}, S_{3}\right) \in \mathcal{W}$ means that if the players of $S_{1}$ vote in favor of a social alternative, the players of $S_{2}$ abstain or are neutral, and the members of $S_{3}$ vote against it, then this alternative will be adopted as the social choice. $\left(S_{1}, S_{2}, S_{3}\right)$ is called a winning partition or a majority. Given a voting game with abstention, assuming that voters $i$ and $j$ have the same initial degree of approval, player $i$ is said to be at least as influential as $j$ if whenever $j$ can transform a loosing partition $\left(S_{1}, S_{2}, S_{3}\right)$ into a winning partition by an upward shift in his level of approval, voter $i$ can achieve the same by an identical shift ceteris paribus. In [31] the authors also compare the influence relation with some generalizations of the Shapley-Shubik and Banzhaf-Coleman power indices to voting games with abstention $[11,15,16]$.

The present paper is a continuation of our research on the influence indices initiated in [20]. We aim at enlarging the set of possible yes-no decisions to a multi-choice framework, and at investigating the generalized influence indices and other tools related to the influence in the multi-choice model. In fact, we escape now from a voting situation, where players have to say either 'yes' or 'no'. It is assumed that each player has a totally ordered set of possible actions, the same for each player, and he has to choose one of the actions. A player has an inclination to choose a particular action, but his decision may be different from the inclination, due to the influence by other players. Our aim is to investigate a general framework of influence which can model many decision situations. Hence, the set of possible actions can be, for instance, yes-no-abstain, like for ternary voting games, a yes-no model with intermediate opinions like 'maybe no', 'don't know', 'maybe yes', etc., or levels of participation/involvement, like in multi-choice games. In our model, there is no outside event which could make a player change his decision: only interactions between players take place. Consequently, if a player decides differently from his original inclination, it is considered as the unique result of the influence between players. First, we analyze a positive influence, which measures how much a coalition attracts a player, i.e., pulls the player's vote near to the inclination of the coalition. A player who has an inclination different from the inclination of a given coalition is said to be influenced by this coalition if his decision is closer to the inclination of the coalition than his inclination was. A direct influence in the yes-no model, which was analyzed in [20], is a particular case of the positive influence: the inclinations of the player and the coalition are different from each other, but the decision of the player coincides with the inclination of the coalition. We also investigate a negative influence. For each inclination vector in which the members of a given coalition have the same inclination, there is one (or two) action(s) which is (are) the most extreme action(s). These actions lie farthest from the inclination of the coalition. If the inclination of a player is different from such actions, and his decision comes 'closer' to the extreme action, we say that there is a negative influence of the coalition on the player. An opposite influence in the yes-no model, which was investigated in [20], is a particular case of the negative influence: the inclinations of the player and the coalition are the same, but the decision of the player is different from the inclination of the coalition. 
In the set of possible actions, we display a set of 'neutral actions' defined as follows: a coalition with the inclination to choose the neutral action has no ability to influence a player. Hence, if a player decides differently from his inclination, it is assumed to be NOT due to the influence of a coalition with the 'neutral inclination'. One particular case of the three-action model is a generalization of the original yes-no framework to a yes-no-abstention scheme, where each player has an inclination to say either 'yes' (action +1 ), or 'no' (action -1), or to abstain (action 0). The abstention may be defined as such a neutral action. It is then assumed that a coalition of abstaining players has no influence ability, but the abstention may be the result of the influence.

In the paper, we consider the set of followers, where by a follower of a given coalition of players we mean, roughly speaking, the agent whose decision is always closer to the inclination of the coalition in question than his inclination was. A perfect follower of a coalition is a player who always decides according to the inclination of the coalition in question. We study properties of the sets of followers and perfect followers, and compare the results with the ones concerning the followers in the yes-no model. We also define several influence functions defined in the multi-choice model, and study their properties. We consider the majority function, the guru function, the identity function, and the mass psychology function. Similar to the case of the followers, we compare the results on the influence functions defined for the multi-choice model with the results on the analogous influence functions introduced in the yes-no model of influence.

The structure of the paper is the following. In Section 2, the generalized influence indices are defined and studied. For simplicity reasons we do not add the word generalized when defining and discussing the indices, but they all measure the influence in a more general framework, i.e., in the multi-choice model, and they are generalized versions of the measures considered in [20]. In Section 3, we study the concepts of a follower, a perfect follower of a coalition, and the concept of a purely influential function. Different influence functions and their properties are investigated in Section 4. In Section 5 we conclude. We also present several examples.

\section{The generalized influence indices}

The general framework is the following. We consider a social network with the set of players (agents, actors) denoted by $N=\{1, \ldots, n\}$. There is a totally ordered set of possible actions denoted by $\mathcal{A}$, which is finite, and contains at least two actions. To each action in $\mathcal{A}$ a real number is assigned, so that the ordering of these numbers reflect the ordering of the actions (ordinal scale). Let us denote by $A$ the set of these numbers. Assuming there are no two actions with the same rank, we have a bijection between $\mathcal{A}$ and $A$, so that we can deal only with $A$. Let us give some typical examples.

(i) The simplest case is to consider a voting situation with two actions 'yes' and 'no'. This is the model we developed in [20], and these actions were coded by the numbers 1 and -1 respectively.

(ii) A useful improvement of the previous example is to allow abstention as a possible action. This could be called the yes-no-abstention model, and refers to ternary voting games proposed by Felsenthal and Machover [11]. A natural coding of these actions could be $-1,0,1$, with 0 denoting abstention. 
(iii) Considering again a voting situation, we may allow voters to express less clear-cut opinions, and let them choose among: \{no, maybe no, don't know, maybe yes, yes $\}$, or any similar set of actions like: \{false, maybe false, ..., true\}, \{strongly disagree, disagree, slightly disagree,..., strongly agree\}, \{strong rejection, rejection, weak rejection, borderline, ..., strong acceptance\}, etc., which are common in opinion polls, questionnaires on the web, evaluation grids, etc. Coding these actions may be done by using integers, using 0 for the action demarcating the two tendencies (yes/no, true/false, agree/disagree, etc.).

(iv) The last situation is inspired by multichoice games [22], where each player has to choose a level of participation or degree of involvement in the game. For example, this could be the amount of funding given to some charitable or humanitary institution, some country in the Third World, or some area hit by a natural disaster, etc. In this case, the simplest coding is to use the amount of funding itself.

Remark 1 With each example we have given a possible "natural" coding of the actions. In its full generality, coding abstract elements by numbers refers to measurement theory, and is not a simple problem. As it will be explained in the following, our model basically computes degrees of influence and follower functions. The first notion is a cardinal notion, which needs an interval scale to be meaningful. That is, the coding given to the actions should reflect only a distance between the actions, and the zero is arbitrary. In other words, the coding is unique up to a positive affine transformation. For example, in the yes-no model, the numbers 1, 0 could be used as well. On the other hand, the second notion (follower function) is purely ordinal, so that any coding consistent with the ordering of the actions is suitable (ordinal scale).

Each player has an inclination to choose one of the actions. Hence, by the inclination of a player we mean the action the player wants to choose. An inclination vector, denoted by $i$, is an $n$-vector consisting of the actions that the players are inclined to choose. Let $I$ be the set of all inclination vectors, that is, $I=A^{n}$ using the bijection between $\mathcal{A}$ and $A$. It is assumed that players may influence each others, and due to the influences in the network, the final decision of a player may be different from his original inclination. In other words, each inclination vector $i \in I$ is transformed into a decision vector $B i$, where $B: I \rightarrow I, i \mapsto B i$ is the influence function. The set of all influence functions will be denoted by $\mathcal{B}$. The decision vector $B i$ is an $n$-vector consisting of the decisions made by the players.

In the set of all actions $\mathcal{A}$, we distinguish the subset $\mathcal{A}^{0} \subset \mathcal{A}$, possibly empty, which is the set of all neutral actions. A neutral action is defined as an action such that a coalition with the inclination to choose the neutral action has no ability to influence a player. Note that however, neutral action may be the result of the influence. We denote by $A^{0}$ the corresponding set of numerical values. Let us consider for example the yes-no-abstention model, coded by $\{-1,0,1\}$. If we assume that an abstaining coalition has no ability to influence the players, then $A^{0}=\{0\}$. On the other hand, if a coalition with the inclination to abstain is assumed to be able to influence a player, then $A^{0}=\emptyset$.

Before formalizing the influence concepts, we introduce several notations for convenience. First of all, cardinality of sets $S, T, \ldots$ will be denoted by the corresponding lower case $s, t, \ldots$ We omit braces for sets, e.g., $\{k, m\}, N \backslash\{j\}, S \cup\{j\}$ will be written $k m$, 
$N \backslash j, S \cup j$, etc. We introduce for any $\emptyset \neq S \subseteq N$

$$
I_{S}:=\left\{i \in I \mid \forall k, j \in S\left[i_{k}=i_{j} \wedge i_{k} \in A \backslash A^{0}\right]\right\},
$$

which is simply the set of inclination vectors under which all players in $S$ have the inclination to choose the same action, different from the neutral action. We denote by $i_{S} \in A \backslash A^{0}$ the value $i_{k}$ for some $k \in S, i \in I_{S}$, that is, the inclination of the members of $S$ under $i$.

Let us now focus on the influence issue. In Sections 2.1 and 2.2, we propose how to measure the positive influence and the negative influence, respectively.

\subsection{The positive influence indices}

The concept of the positive influence adopted in this section is related to the concept of the direct influence of a coalition on a player defined in [20]. It is assumed that the player's inclination is different from the inclination of the coalition, and the player's decision differs from his inclination. However, in the yes-no framework, if a player votes differently from his inclination, the change is uniquely defined, since there are only two possibilities: 'yes' or 'no'. In the model with an ordered set of possible actions, we can observe more than just the changes, i.e., we have also an information about different distances between actions. Consequently, in the generalized model with an ordered set of possible actions, we need to introduce a more sophisticated measure of the influence, which distinguishes 'how much' a player changes his 'position'.

Let for each $S \subseteq N$ and $j \in N \backslash S$

$$
I_{S \rightarrow j}:=\left\{i \in I_{S} \mid i_{j} \neq i_{S}\right\} .
$$

$I_{S \rightarrow j}$ denotes the set of all inclination vectors of potential positive influence of $S$ on $j$. Given coalition $S \subset N$, player $j \notin S$, and inclination vector $i \in I_{S \rightarrow j}$, there is a certain (positive) distance $\left|i_{j}-i_{S}\right|$ between $i_{j}$ and $i_{S}$. Under the influence, the decision $(B i)_{j}$ of player $j$ may be different from his inclination, and what we can also measure is a distance $\left|(B i)_{j}-i_{S}\right|$ between the decision of the player and the inclination of the coalition. Did the coalition succeed in making the player 'put' his decision closer to the inclination of the coalition? Consequently, for each $S \subseteq N, j \in N \backslash S$, and $B \in \mathcal{B}$, we can define the set of all inclination vectors of positive influence of $S$ on $j$ under given $B$ as

$$
I_{S \rightarrow j}^{\text {pos }}(B):=\left\{i \in I_{S \rightarrow j}||(B i)_{j}-i_{S}|<| i_{j}-i_{S} \mid\right\} .
$$

For each $S \subseteq N, j \in N \backslash S$ and $i \in I_{S \rightarrow j}$, we introduce a weight $\alpha_{i}^{S \rightarrow j} \in[0,1]$ of influence of coalition $S$ on $j \in N \backslash S$ under the inclination vector $i \in I_{S \rightarrow j}$. We assume that for each $S \subseteq N$ and $j \in N \backslash S$, there exists $i \in I_{S \rightarrow j}$ such that $\alpha_{i}^{S \rightarrow j}>0$. Moreover, we impose a kind of symmetry assumption that $\alpha_{i}^{S \rightarrow j}$ depends solely on numbers $n^{a}(S, j, i)$, for $a \in A \backslash\left(A^{0} \cup\left\{i_{j}\right\}\right)$, where $n^{a}(S, j, i)$ is the number of players in $N \backslash j$ with the inclination to choose action $a \in A \backslash\left(A^{0} \cup\left\{i_{j}\right\}\right)$ under $i \in I_{S \rightarrow j}$.

Definition 1 Given $B \in \mathcal{B}$, for each $S \subseteq N, j \in N \backslash S$, the weighted positive influence index of coalition $S$ on player $j$ is defined as

$$
D_{\alpha}(B, S \rightarrow j):=\frac{\sum_{i \in I_{S \rightarrow j}^{\text {pos }}(B)}\left[\left|i_{j}-i_{S}\right|-\left|(B i)_{j}-i_{S}\right|\right] \alpha_{i}^{S \rightarrow j}}{\sum_{i \in I_{S \rightarrow j}}\left|i_{j}-i_{S}\right| \alpha_{i}^{S \rightarrow j}} \in[0,1] .
$$


The possibility positive influence index of coalition $S$ on player $j$ is given by

$$
\bar{D}(B, S \rightarrow j):=\frac{\sum_{i \in I_{S \rightarrow j}^{p o s}(B)}\left[\left|i_{j}-i_{S}\right|-\left|(B i)_{j}-i_{S}\right|\right]}{\sum_{i \in I_{S \rightarrow j}}\left|i_{j}-i_{S}\right|},
$$

that is,

$$
\begin{gathered}
\bar{D}(B, S \rightarrow j)=D_{\bar{\alpha}}(B, S \rightarrow j) \\
\text { where } \bar{\alpha}_{i}^{S \rightarrow j}=1 \text { for each } i \in I_{S \rightarrow j} .
\end{gathered}
$$

The weighted positive influence index defined in (4) generalizes the weighted direct influence index defined for the yes-no model of influence (see [20], Definition 5). Similarly, the possibility positive influence index defined in (5) is a generalization of the possibility direct influence index for the yes-no model (see [20], Definition 3).

Remark 2 Given $B \in \mathcal{B}, S \subseteq N, j \in N \backslash S$, if for each $i \in I_{S \rightarrow j}^{\text {pos }}(B)$, the decision of player $j$ 'lies' between his inclination and the inclination of coalition $S$, i.e., if for each $i \in I_{S \rightarrow j}^{p o s}(B)$

$$
\left|i_{j}-i_{S}\right|=\left|(B i)_{j}-i_{j}\right|+\left|(B i)_{j}-i_{S}\right|
$$

then the weighted positive influence index of coalition $S$ on player $j$ is equal to

$$
D_{\alpha}(B, S \rightarrow j)=\frac{\sum_{i \in I_{S \rightarrow j}^{\text {pos }}(B)}\left|(B i)_{j}-i_{j}\right| \alpha_{i}^{S \rightarrow j}}{\sum_{i \in I_{S \rightarrow j}}\left|i_{j}-i_{S}\right| \alpha_{i}^{S \rightarrow j}} .
$$

Note that we do not distinguish between two situations in which the decisions of the influenced player are different from each other, but their distances from the inclination of the coalition are the same. Let us consider a situation, in which the decision of the influenced player 'lies' between his inclination and the inclination of the coalition. Next, let us imagine that the influenced player 'puts' his decision on the 'other side' of the inclination of the coalition, farther from his own inclination, but the distance between his decision and the inclination of the coalition is the same as before. If this holds for each $i \in I_{S \rightarrow j}^{p o s}(B)$, we get the same weighted positive influence index of the coalition on the player.

If we wanted to register only the fact of the influence between players, that is, to consider all cases when a player's decision is different from his inclination, without distinguishing how much the player changes his position, then we could define the general influence index:

$$
\bar{d}(B, S \rightarrow j):=\frac{\left|I_{S \rightarrow j}^{*}(B)\right|}{\left|I_{S \rightarrow j}\right|}
$$

where $I_{S \rightarrow j}$ is defined in (2), and

$$
I_{S \rightarrow j}^{*}(B):=\left\{i \in I_{S \rightarrow j} \mid(B i)_{j} \neq i_{j}\right\} .
$$

In the yes-no framework, $\bar{d}(B, S \rightarrow j)$ coincides with the possibility direct influence index defined in [20]. Note that

Fact 1 Given $B \in \mathcal{B}, S \subseteq N, j \in N \backslash S$, if $\bar{D}(B, S \rightarrow j)=1$, then $\bar{d}(B, S \rightarrow j)=1$. 
Proof: Suppose $\bar{D}(B, S \rightarrow j)=1$. This means that

$$
\sum_{i \in I_{S \rightarrow j}^{\text {pos }}(B)}\left[\left|i_{j}-i_{S}\right|-\left|(B i)_{j}-i_{S}\right|\right]=\sum_{i \in I_{S \rightarrow j}}\left|i_{j}-i_{S}\right|
$$

and this implies that $I_{S \rightarrow j}^{\text {pos }}(B)=I_{S \rightarrow j}$ and $(B i)_{j}=i_{S}$ for all $i \in I_{S \rightarrow j}$. Hence, $(B i)_{j} \neq i_{j}$ always holds.

\subsection{The negative influence indices}

The concept of the influence adopted in this section is related to the opposite influence of a coalition on a player in the yes-no model defined in [20]. Under the opposite influence in the yes-no model, the inclinations of the coalition and the player coincide, but the decision of the player differs from his inclination. When measuring a negative influence of a coalition on a player in the model with an ordered set of possible actions, we look for the inclination vectors, in which the inclination of the player is different from the action(s) placed farthest from the inclination of the coalition. If the decision of the player is farther from the inclination of the coalition than his inclination was, we say that such an inclination vector is the inclination vector of (observed) negative influence of the coalition on the player. Let us note that while in the case of the positive influence, the coalition tries to 'attract' the player's decision as close to its incli! nation as possible, when considering the negative influence, the decision of the player is as far from the inclination of the coalition as possible. Below, we formalize the concepts of negative influence. For each $a \in A \backslash A^{0}$, we define

$$
M(a):=\left\{\widetilde{a} \in A\left|\widetilde{a}=\arg \max _{a^{\prime} \in A}\right| a-a^{\prime} \mid\right\} .
$$

$M(a)$ is the set of all actions whose distance to the action $a$ is maximal. Note that a neutral action may belong to the set $M(a)$. Of course, $|M(a)| \in\{1,2\}$. In particular, for each $S \subseteq N$ and $i \in I_{S}$, the coalition $S$ has its inclination $i_{S}$, and then $M\left(i_{S}\right)$ is the set of all possible actions which are the farthest actions from the inclination $i_{S}$, and $M\left(i_{S}\right)$ may have either 1 or 2 elements. For each $S \subseteq N$ and $j \in N \backslash S$, the set of all inclination vectors of potential negative influence of $S$ on $j$ is defined as

$$
\widetilde{I}_{S \rightarrow j}:=\left\{i \in I_{S} \mid i_{j} \notin M\left(i_{S}\right)\right\} .
$$

For each $S \subseteq N, j \in N \backslash S, B \in \mathcal{B}$, and $i \in \widetilde{I}_{S \rightarrow j}$

$$
\begin{gathered}
\widetilde{i}_{S}^{i_{j}}:=\arg \min _{\widetilde{i}_{S} \in M\left(i_{S}\right)}\left|i_{j}-\widetilde{i}_{S}\right| \\
\widetilde{i}_{S}^{(B i)_{j}}:=\arg \min _{\widetilde{i}_{S} \in M\left(i_{S}\right)}\left|(B i)_{j}-\widetilde{i}_{S}\right| .
\end{gathered}
$$

If $\left|M\left(i_{S}\right)\right|=2$, then $\widetilde{i}_{S}^{i_{j}}$ is the action from $M\left(i_{S}\right)$ which is closer to the inclination $i_{j}$ of player $j$, and $\widetilde{i}_{S}^{(B i)_{j}}$ is the action from $M\left(i_{S}\right)$ which is closer to the decision $(B i)_{j}$ of $j$. 
Of course, if $\left|M\left(i_{S}\right)\right|=1$, then $\widetilde{i}_{S}^{i_{j}}=\widetilde{i}_{S}^{(B i)_{j}}$. The set of all inclination vectors of negative influence of $S$ on $j$ under given $B$ is defined as

$$
\widetilde{I}_{S \rightarrow j}^{n e g}(B):=\left\{i \in \widetilde{I}_{S \rightarrow j}|| i_{j}-\widetilde{i}_{S}^{i_{j}}|>|(B i)_{j}-\widetilde{i}_{S}^{(B i)_{j}} \mid\right\} .
$$

In a similar way as before, for each $S \subseteq N, j \in N \backslash S$ and $i \in \widetilde{I}_{S \rightarrow j}$, we introduce a weight $\alpha_{i}^{S \rightarrow j} \in[0,1]$ of influence of coalition $S$ on $j \in N \backslash S$ under the inclination vector $i \in \widetilde{I}_{S \rightarrow j}$, where $\alpha_{i}^{S \rightarrow j}$ depends on numbers $n^{a}(S, j, i)$, for $a \in A \backslash A^{0}$.

Definition 2 Given $B \in \mathcal{B}$, for each $S \subseteq N, j \in N \backslash S$, the weighted negative influence index of coalition $S$ on player $j$ is defined as

$$
D_{\alpha}^{n e g}(B, S \rightarrow j):=\frac{\sum_{i \in \widetilde{I}_{S \rightarrow j}^{\text {neg }}(B)}\left[\left|i_{j}-\widetilde{i}_{S}^{i_{j}}\right|-\left|(B i)_{j}-\widetilde{i}_{S}^{(B i)_{j}}\right|\right] \alpha_{i}^{S \rightarrow j}}{\sum_{i \in \widetilde{I}_{S \rightarrow j}}\left|i_{j}-\widetilde{i}_{S}^{i j}\right| \alpha_{i}^{S \rightarrow j}} \in[0,1] .
$$

The possibility negative influence index of coalition $S$ on player $j$ is given by

$$
\bar{D}^{n e g}(B, S \rightarrow j):=\frac{\sum_{i \in \widetilde{I}_{S \rightarrow j}^{n e g}(B)}\left[\left|i_{j}-\widetilde{i}_{S}^{i_{j}}\right|-\left|(B i)_{j}-\widetilde{i}_{S}^{(B i)_{j}}\right|\right]}{\sum_{i \in \widetilde{I}_{S \rightarrow j}}\left|i_{j}-\widetilde{i}_{S}^{i j}\right|} .
$$

The weighted negative influence index defined in (15) generalizes the weighted opposite influence index defined for the yes-no model of influence (see [20], Definition 5). Similarly, the possibility negative influence index defined in (16) is a generalization of the possibility opposite influence index for the yes-no model (see [20], Definition 3).

\subsection{The Examples}

The typical situations given in the beginning of Section 2 show a broad applicability of the generalized framework and the influence indices that are studied in the paper. One of the natural applications of the model with an ordered set of actions is an application to politics, in particular to elections. An ideological line indicating a political orientation of candidates or parties, from the Left Wing via the Center to the Right Wing, has its natural interpretation here. Below, we present two other examples, corresponding respectively to situations (ii) and (iv) of Section 2, in which we calculate all concepts introduced in the paper.

Example 1 As mentioned before, a particular case of our generalized framework is a yes-no-abstention model, in which each player has an inclination either to say 'yes' or 'no', or to abstain. Let us check how the concepts and formulas introduced above look like for this three-action example. We have $A=\{-1,0,+1\}, A^{0}=\{0\}$,

$$
\begin{gathered}
I_{S}=\left\{i \in I \mid \forall k, j \in S\left[i_{k}=i_{j} \wedge i_{k} \neq 0\right\}\right. \\
I_{S \rightarrow j}=\left\{i \in I_{S} \mid i_{j} \neq i_{S}\right\} \\
I_{S \rightarrow j}^{*}(B)=\left\{i \in I_{S \rightarrow j} \mid(B i)_{j} \neq i_{j}\right\}
\end{gathered}
$$


$\alpha_{i}^{S \rightarrow j}$ depends on the number $n^{i_{S}}(S, j, i)$, where

$$
n^{i_{S}}(S, j, i)=\left|\left\{m \in N \backslash j \mid i_{m}=i_{S}\right\}\right| .
$$

Let for $a \in\{-1,+1\}, b, c \in\{-1,0,+1\}$, and $x \in\{a, b, c\}, s(x)$ denote the sign of $x$, that is, $s(x)=+$ if $x=+1, s(x)=-$ if $x=-1$, and $s(x)=0$ if $x=0$. Moreover, let

$$
I_{S \rightarrow j}^{s(a) s(b) s(c)}(B):=\left\{i \in I_{S} \mid i_{S}=a \wedge i_{j}=b \wedge(B i)_{j}=c\right\}
$$

Then we have

$$
\begin{gathered}
\bar{D}(B, S \rightarrow j)= \\
\frac{\left|I_{S \rightarrow j}^{-0-}(B)\right|+\left|I_{S \rightarrow j}^{-+0}(B)\right|+\left|I_{S \rightarrow j}^{+0+}(B)\right|+\left|I_{S \rightarrow j}^{+-0}(B)\right|+2\left(\left|I_{S \rightarrow j}^{-+-}(B)\right|+\left|I_{S \rightarrow j}^{+-+}(B)\right|\right)}{2 \cdot 3^{n-s}} \\
\bar{d}(B, S \rightarrow j)=\frac{\left|I_{S \rightarrow j}^{*}(B)\right|}{\left|I_{S \rightarrow j}\right|}=\frac{\left|I_{S \rightarrow j}^{-0-}(B)\right|+\left|I_{S \rightarrow j}^{-+0}(B)\right|+\left|I_{S \rightarrow j}^{+0+}(B)\right|+\left|I_{S \rightarrow j}^{+-0}(B)\right|}{4 \cdot 3^{n-s-1}}+ \\
+\frac{\left|I_{S \rightarrow j}^{-+-}(B)\right|+\left|I_{S \rightarrow j}^{+-+}(B)\right|+\left|I_{S \rightarrow j}^{-0+}(B)\right|+\left|I_{S \rightarrow j}^{+0-}(B)\right|}{4 \cdot 3^{n-s-1}} .
\end{gathered}
$$

For $a \in\{-1,+1\}, M(a)=\{-a\}$

$$
\begin{gathered}
\widetilde{I}_{S \rightarrow j}=\left\{i \in I_{S}|| i_{j}-i_{S} \mid \in\{0,1\}\right\} \\
\widetilde{i}_{S}^{i_{j}}=\widetilde{i}_{S}^{(B i)_{j}}=-i_{S} \\
\widetilde{I}_{S \rightarrow j}^{n e g}(B)=\left\{i \in I_{S}|| i_{j}+i_{S}|>|(B i)_{j}+i_{S} \mid\right\} \\
\bar{D}^{n e g}(B, S \rightarrow j)= \\
\frac{\left|I_{S \rightarrow j}^{-0+}(B)\right|+\left|I_{S \rightarrow j}^{--0}(B)\right|+\left|I_{S \rightarrow j}^{+0-}(B)\right|+\left|I_{S \rightarrow j}^{++0}(B)\right|+2\left(\left|I_{S \rightarrow j}^{--+}(B)\right|+\left|I_{S \rightarrow j}^{++-}(B)\right|\right)}{2 \cdot 3^{n-s}} .
\end{gathered}
$$

Example 2 Let us consider another very simple example with three players and three actions. A three-member committee of the research lab is to decide about a new budget for external seminars. The actions are placed on the line 'changing the seminar budget':

- Action $a$ : to decrease the present seminar budget by 1000 euro;

- Action $b$ : to keep the seminar budget unchanged;

- Action $c$ : to increase the present seminar budget by 2000 euro.

According to Remark 1, we may code these actions by $-1,0$ and +2 respectively.

Determining the seminar budget is not an obvious decision to be made, because it may be related to a change of the budget for other expenses of the lab. The committee consists of the director of the lab (player 1), and two other professors who chair two different sections. Each committee member has an inclination to choose one of the actions, but due to some influence of the others, he can vote differently from his preliminary intention. It is assumed that the director of the lab always votes according to his (original) inclination. If the director and another professor both want to change the budget, the remaining colleague will follow their inclination. The boss influences on his own a colleague with the inclination to keep the seminar budget unchanged, but if a coalition of the committee 
members is inclined to keep the old budget, it has no ability to influence the remaining professor(s).

Using the notation introduced in the paper, we have the committee (the set of players) $N=\{1,2,3\}$, the set of actions $A=\{-1,0,+2\}$, and the action 0 as the neutral action, $A^{0}=\{0\}$. Moreover, there are 27 possible inclination vectors, $|I|=27$. Table 1 shows the inclination vectors and the decision vectors.

Table 1. The inclination and decision vectors

\begin{tabular}{c|c||c|c||c|c}
$i \in I$ & $B i$ & $i \in I$ & $B i$ & $i \in I$ & $B i$ \\
\hline \hline$(-1,-1,-1)$ & $(-1,-1,-1)$ & $(0,0,0)$ & $(0,0,0)$ & $(2,2,2)$ & $(2,2,2)$ \\
\hline$(-1,-1,0)$ & $(-1,-1,-1)$ & $(0,0,-1)$ & $(0,0,-1)$ & $(2,2,-1)$ & $(2,2,2)$ \\
\hline$(-1,0,-1)$ & $(-1,-1,-1)$ & $(0,-1,0)$ & $(0,-1,0)$ & $(2,-1,2)$ & $(2,2,2)$ \\
\hline$(0,-1,-1)$ & $(0,-1,-1)$ & $(-1,0,0)$ & $(-1,-1,-1)$ & $(-1,2,2)$ & $(-1,2,2)$ \\
\hline$(-1,-1,2)$ & $(-1,-1,-1)$ & $(0,0,2)$ & $(0,0,2)$ & $(2,2,0)$ & $(2,2,2)$ \\
\hline$(-1,2,-1)$ & $(-1,-1,-1)$ & $(0,2,0)$ & $(0,2,0)$ & $(2,0,2)$ & $(2,2,2)$ \\
\hline$(2,-1,-1)$ & $(2,-1,-1)$ & $(2,0,0)$ & $(2,2,2)$ & $(0,2,2)$ & $(0,2,2)$ \\
\hline$(-1,0,2)$ & $(-1,-1,2)$ & $(0,-1,2)$ & $(0,-1,2)$ & $(2,-1,0)$ & $(2,-1,2)$ \\
\hline$(-1,2,0)$ & $(-1,2,-1)$ & $(0,2,-1)$ & $(0,2,-1)$ & $(2,0,-1)$ & $(2,2,-1)$
\end{tabular}

Note that for $S \subseteq\{2,3\}, D_{\alpha}(B, S \rightarrow 1)=0$, because $(B i)_{1}=i_{1}$ for each $i \in I$. The possibility positive and general influence indices are as follows:

$$
\begin{gathered}
\bar{D}(B, 1 \rightarrow 2)=\bar{D}(B, 1 \rightarrow 3)=\frac{5}{9}, \quad \bar{d}(B, 1 \rightarrow 2)=\bar{d}(B, 1 \rightarrow 3)=\frac{2}{3} \\
\bar{D}(B, 2 \rightarrow 3)=\bar{D}(B, 3 \rightarrow 2)=\bar{d}(B, 2 \rightarrow 3)=\bar{d}(B, 3 \rightarrow 2)=\frac{1}{3} \\
\bar{D}(B, 12 \rightarrow 3)=\bar{D}(B, 13 \rightarrow 2)=\bar{d}(B, 12 \rightarrow 3)=\bar{d}(B, 13 \rightarrow 2)=1 .
\end{gathered}
$$

When considering, for instance, the influence of player 1 on player 2 , we verify 12 inclination vectors in which player 1's inclination is both different from 0 and different from player 2's inclination. In the case of 8 out of these 12 inclination vectors, player 2 votes differently from his inclination. Note however that the influence has different strength. For instance, when we look at the inclination vectors $(-1,0,-1)$ and $(-1,2,-1)$, in both cases the decision vector is equal to $(-1,-1,-1)$. Nevertheless, in the first case, player 2 has moved only from 0 to -1 , while in the second case, he changed from 2 to -1 .

We calculate also the possibility negative influence indices which, in most cases except two indices, are equal to 0 . These indices with positive values are:

$$
\bar{D}^{\text {neg }}(B, 2 \rightarrow 3)=\bar{D}^{\text {neg }}(B, 3 \rightarrow 2)=\frac{(1-0)+(2-0)}{27}=\frac{1}{9} .
$$

For instance, the inclination vector $(-1,2,0)$ leads to the decision vector $(-1,2,-1)$, which counts for the negative influence of player 2 on player 3 . Both in the denominator and the numerator of $\bar{D}^{n e g}(B, 2 \rightarrow 3)$ we write 1 : the farthest action of player 2 with the inclination 2 is -1 , which is of a distance equal to 1 from the neutral inclination of player 3 , and player 3 decides for the action -1 . Moreover, the inclination vector 
$(2,-1,0)$ leads to the decision vector $(2,-1,2)$, and we write 2 both in the denominator and the numerator of $\bar{D}^{\text {neg }}(B, 2 \rightarrow 3)$. Nevertheless, we should be aware that although the negative influence index of player 2 on player 3 has a positive value, it is rather due to the positive influence of player 1 on player 3 . As it was described before, player 1 has the ability to make an abstaining player decide according to the inclination of player 1. In both inclination vectors $(-1,2,0)$ and $(2,-1,0)$, the inclinations of players 1 and 2 are as far from each other as possible. In a similar way we can analyze the case of $\bar{D}^{n e g}(B, 3 \rightarrow 2)$.

\section{Following a coalition}

In this section, we focus on the positive influence. Concepts related to the positive influence are the concepts of follower and of perfect follower of a given coalition. As mentioned in the Introduction, a follower of a given coalition of players is an agent whose decision is never farther from the inclination of the coalition in question than his inclination was. A voter who always decides according to the inclination of the coalition in question is called a perfect follower of that coalition.

Definition 3 Let $\emptyset \neq S \subseteq N$ and $B \in \mathcal{B}$. The set of followers of $S$ under $B$ is defined as

$F_{B}(S):=\left\{j \in N \mid \forall i \in I_{S}\left[\left[i_{j} \neq i_{S} \Rightarrow\left|(B i)_{j}-i_{S}\right|<\left|i_{j}-i_{S}\right|\right] \wedge\left[i_{j}=i_{S} \Rightarrow(B i)_{j}=i_{S}\right]\right]\right\}$,

where $F_{B}(\emptyset):=\emptyset$, and the set of perfect followers of $S$ under $B$ is defined as

$$
F_{B}^{\text {per }}(S):=\left\{j \in N \mid \forall i \in I_{S}\left[(B i)_{j}=i_{S}\right]\right\}
$$

Of course, each perfect follower is also a follower, i.e., for each $B \in \mathcal{B}$ and $S \subseteq N$,

$$
F_{B}^{\text {per }}(S) \subseteq F_{B}(S)
$$

Note that $F_{B}$ and $F_{B}^{\text {per }}$ define functions from $2^{N}$ to $2^{N}$, which we call follower function and perfect follower function. The next proposition studies the properties of these functions.

Proposition 1 Let $B \in \mathcal{B}$. Then the following holds:

(i) Whenever $S \cap T=\emptyset, F_{B}(S) \cap F_{B}(T)=\emptyset$.

(ii) $F_{B}$ is an isotone function $\left(S \subseteq S^{\prime}\right.$ implies $\left.F_{B}(S) \subseteq F_{B}\left(S^{\prime}\right)\right)$.

Consequently, if $F_{B}(N)=\emptyset$, then $F_{B} \equiv \emptyset$.

(iii) For each $j \in F_{B}^{\text {per }}(S) \backslash S, D_{\alpha}(B, S \rightarrow j)=1$, and $D_{\alpha}^{\text {neg }}(B, S \rightarrow j)=0$.

Proof: (i) Since $S \cap T=\emptyset, I_{S} \cap I_{T}$ strictly includes $I_{S \cup T}$. Then there exists $i \in I_{S} \cap I_{T}$ such that $i_{S} \neq i_{T}$. Suppose that $j \in F_{B}(S) \cap F_{B}(T)$. Take $i$ such that $i_{j} \neq i_{S}$ and $0<\left|i_{S}-i_{T}\right|=\left|i_{S}-i_{j}\right|+\left|i_{j}-i_{T}\right|$. We have $\left|(B i)_{j}-i_{S}\right|<\left|i_{j}-i_{S}\right|$ and $\left|(B i)_{j}-i_{T}\right| \leq\left|i_{j}-i_{T}\right|$. Hence, $\left|(B i)_{j}-i_{S}\right|+\left|(B i)_{j}-i_{T}\right|<\left|i_{j}-i_{S}\right|+\left|i_{j}-i_{T}\right|=\left|i_{S}-i_{T}\right|$. On the other hand, $\left|i_{S}-i_{T}\right|=\left|i_{S}-(B i)_{j}+(B i)_{j}-i_{T}\right| \leq\left|i_{S}-(B i)_{j}\right|+\left|(B i)_{j}-i_{T}\right|$, a contradiction.

(ii) Take $S \subseteq S^{\prime}$ and $j \in F_{B}(S) . i \in I_{S^{\prime}}$ implies $i \in I_{S}$ by antitonicity. If $i_{j} \neq i_{S}$, then $\left|(B i)_{j}-i_{S}\right|<\left|i_{j}-i_{S}\right|$, and if $i_{j}=i_{S}$, then $(B i)_{j}=i_{S}$. Since $i_{S}=i_{S^{\prime}}, j \in F_{B}\left(S^{\prime}\right)$. 
(iii) Let $B \in \mathcal{B}, S \subset N, F_{B}^{\text {per }}(S) \neq \emptyset$, and $j \in F_{B}^{\text {per }}(S) \backslash S$. Hence, for each $i \in I_{S}$, $(B i)_{j}=i_{S}$, and therefore $D_{\alpha}(B, S \rightarrow j)=1$, and $D_{\alpha}^{\text {neg }}(B, S \rightarrow j)=0$.

Note that Proposition 1 generalizes our results presented in [20] to the model with an ordered set of possible actions. In [20] (Prop. 2), we prove similar results (i) and (ii) for the set of followers in the yes-no model, where the concept of follower in such a model coincides with the concept of perfect follower introduced in the present paper. In [20] (Prop. 2(iii)), we show that the weighted direct influence index of a coalition on its follower outside the coalition is equal to 1 , while the weighted opposite influence index is equal to 0 . In the multi-choice game, these properties remain true only for the perfect followers.

Assume $F_{B}$ is not identically the empty set. The kernel of $B$ is the set of "true" influential coalitions:

$$
\mathcal{K}(B):=\left\{S \in 2^{N} \mid F_{B}(S) \neq \emptyset, \text { and } S^{\prime} \subset S \Rightarrow F_{B}\left(S^{\prime}\right)=\emptyset\right\} .
$$

The kernel is well defined due to isotonicity.

Definition 4 Let $S, T$ be two disjoint nonempty subsets of $N$. $B$ is said to be a purely influential function of $S$ upon $T$ if it satisfies for all $i \in I_{S}$ :

$$
(B i)_{j}= \begin{cases}i_{S} & \text { if } j \in T \\ i_{j} & \text { otherwise. }\end{cases}
$$

The set of such functions is denoted $\mathcal{B}_{S \rightarrow T}$.

Note that these functions are arbitrary on $I \backslash I_{S}$. The cardinality of $\mathcal{B}_{S \rightarrow T}$ is

$$
\left|\mathcal{B}_{S \rightarrow T}\right|=|A|^{n\left(|A|^{s}-|A|+\left|A^{0}\right|\right)|A|^{n-s}}
$$

Proposition 2 Let $S, T$ be two disjoint nonempty subsets of $N$. Then the following holds:

(i) For all $B \in \mathcal{B}_{S \rightarrow T}, F_{B}(S) \supseteq S \cup T$, and $F_{B}^{\text {per }}(S)=S \cup T$.

(ii) For each $B \in \mathcal{B}_{S \rightarrow T}$ and $j \in N \backslash S, D_{\alpha}^{\text {neg }}(B, S \rightarrow j)=0$, and

$$
D_{\alpha}(B, S \rightarrow j)= \begin{cases}1 & \text { if } j \in T \\ 0 & \text { if } j \in N \backslash(S \cup T)\end{cases}
$$

Proof: (i) Take $t \in S \cup T$. If $t \in T$, then for any $i \in I_{S},(B i)_{t}=i_{S}$. If $t \in S$, then for any $i \in I_{S},(B i)_{t}=i_{t}=i_{S}$. Hence $t \in F_{B}(S)$. On the other hand, take $t \in F_{B}^{\text {per }}(S)$. Then for any $i \in I_{S},(B i)_{t}=i_{S}$, and hence $t \in S \cup T$.

(ii) Let $B \in \mathcal{B}_{S \rightarrow T}$. Then for each $i \in I_{S},(B i)_{j}=i_{S}$ for $j \in T$, and $(B i)_{j}=i_{j}$ for $j \notin T$. Since $I_{S \rightarrow j} \subset I_{S}$, we have for each $i \in I_{S \rightarrow j},(B i)_{j}=i_{S}$ for $j \in T$, and $(B i)_{j}=i_{j}$ for $j \notin T$. Hence, if $j \in T$, then $D_{\alpha}(B, S \rightarrow j)=1$, and if $j \in N \backslash(S \cup T)$, then $D_{\alpha}(B, S \rightarrow j)=0$. Moreover, for each $j \in N \backslash S, D_{\alpha}^{\text {neg }}(B, S \rightarrow j)=0$.

Proposition 2 generalizes our results shown in [20] (Prop. 3). However, while in the yes-no model the set of followers of coalition $S$ under a purely influential function of $S$ upon $T$ is equal to $S \cup T$, in the multi-choice model only the inclusion remains valid. 
Example 3 The sets of followers and perfect followers for Example 2 presented in Section 2.3 are the following:

$$
\begin{gathered}
F_{B}^{\text {per }}(S)=F_{B}(S) \text { for each } S \subseteq N \\
F_{B}(\emptyset)=F_{B}(2)=F_{B}(3)=\emptyset, \quad F_{B}(1)=\{1\} \\
F_{B}(23)=\{2,3\}, \quad F_{B}(12)=F_{B}(13)=F_{B}(123)=\{1,2,3\}
\end{gathered}
$$

The kernel $\mathcal{K}(B)=\{\{1\},\{2,3\}\}$. Moreover, $B \in \mathcal{B}_{12 \rightarrow 3} \cap \mathcal{B}_{13 \rightarrow 2}$.

\section{The influence functions}

In this section, several influence functions $B \in \mathcal{B}$, originally defined in [20], are generalized for the multi-choice model. We investigate the properties of these functions and compare them with our results on the analogous functions in the yes-no model. In particular, for each influence function analyzed, we determine the follower and perfect follower functions, and the values of the (positive and negative) weighted influence indices.

Some simple examples of influence functions are:

(i) The majority function. Let $n \geq t>\left\lfloor\frac{n}{2}\right\rfloor$, and introduce for any $i \in I$ and $a \in A \backslash A^{0}$, the set

$$
i^{a}:=\left\{k \in N \mid i_{k}=a\right\} .
$$

The majority influence function $\mathrm{Maj}^{[t]} \in \mathcal{B}$ is defined by

$$
\left(\operatorname{Maj}^{[t]} i\right)_{j}:=\left\{\begin{array}{ll}
a, & \text { if } \exists a \in A \backslash A^{0}\left[\left|i^{a}\right| \geq t\right] \\
i_{j}, & \text { otherwise }
\end{array}, \quad \forall i \in I, \quad \forall j \in N .\right.
$$

If a majority of players have an inclination $a$, then all players decide for $a$. If not, each player decides according to his own inclination.

(ii) The guru function. Let $\widetilde{k} \in N$ be a particular player called the guru. The guru influence function Gur $^{[\tilde{k}]} \in \mathcal{B}$ is defined by

$$
\left(\operatorname{Gur}^{[\widetilde{k}]} i\right)_{j}=i_{\widetilde{k}}, \quad \forall i \in I, \quad \forall j \in N
$$

When a guru exists, every player follows always the guru.

(iii) The identity function $\mathrm{Id} \in \mathcal{B}$ is defined by

$$
\operatorname{Id} i=i, \quad \forall i \in I \text {. }
$$

(iv) The mass psychology function. Let $t \in(0, n]$ and $a \in A \backslash A^{0}$. Functions $B \in \mathcal{B}$ satisfying for each $i \in I$

$$
\text { if }\left|i^{a}\right| \geq t \text {, then }(B i)^{a} \supseteq i^{a}
$$

are called mass psychology influence functions. If there is a sufficiently high number of players with inclination $a$, none of these players will decide differently than $a$, and they will possibly attract other players to choose action $a$. We denote by $\mathcal{B}^{[a, t]}$ the set of such influence functions. 
We list some basic properties of the influence functions mentioned.

Proposition 3 Let $n \geq t>\left\lfloor\frac{n}{2}\right\rfloor$ and consider the majority function Maj ${ }^{[t]}$. Then the following holds:

(i) For each $\emptyset \neq S \subseteq N$ such that $s \geq t$, and for each $j \in N \backslash S, D_{\alpha}\left(\mathrm{Maj}^{[t]}, S \rightarrow j\right)=1$, and $D_{\alpha}^{\text {neg }}\left(\mathrm{Maj}^{[t]}, S \rightarrow j\right)=0$.

If $t=n$, then for each $\emptyset \neq S \subseteq N$ and $j \in N \backslash S$, $D_{\alpha}\left(\mathrm{Maj}^{[t]}, S \rightarrow j\right)=D_{\alpha}^{n e g}\left(\mathrm{Maj}^{[t]}, S \rightarrow j\right)=0$.

(ii) For each $S \subseteq N$,

$$
F_{\mathrm{Maj}}[t](S)= \begin{cases}N, & \text { if } s \geq t \\ S, & \text { if } n-t<s<t \\ \emptyset, & \text { if } s \leq n-t .\end{cases}
$$

(iii) The kernel is $\mathcal{K}\left(\mathrm{Maj}^{[t]}\right)=\{S \subseteq N|| S \mid=n-t+1\}$.

Proof: (i) Let $\emptyset \neq S \subseteq N$ be such that $s \geq t$, and $j \in N \backslash S$. For each $i \in I_{S}$, there is $a \in A \backslash A^{0}$ such that $i_{S}=a$, and therefore $\left|i^{a}\right| \geq s \geq t$. Hence, $\left(\operatorname{Maj}^{[t]} i\right)_{j}=a=i_{S}$ for each $i \in I_{S}$, which gives $D_{\alpha}\left(\mathrm{Maj}^{[t]}, S \rightarrow j\right)=1$, and $D_{\alpha}^{n e g}\left(\mathrm{Maj}^{[t]}, S \rightarrow j\right)=0$.

If $t=n$, then $\left(\mathrm{Maj}^{[t]} i\right)_{j}=i_{j}$ for each $i \in I, j \in N$. Hence, by virtue of (4) and (15), $D_{\alpha}\left(\mathrm{Maj}^{[t]}, S \rightarrow j\right)=0$ and $D_{\alpha}^{\text {neg }}\left(\mathrm{Maj}^{[t]}, S \rightarrow j\right)=0$.

(ii) Let $S \subseteq N$ be such that $s \geq t$. Suppose that $F_{\text {Maj }[t]}(S) \neq N$. Then there exists $j \in N$ such that $j \notin F_{\text {Maj }[t]}(S)$. Hence, there is $\widetilde{i} \in I_{S}$ such that either $\left(\operatorname{Maj}{ }^{[t]} \tilde{i}\right)_{j} \neq \widetilde{i}_{S}$ and $\widetilde{i}_{j}=\widetilde{i}_{S}$, or $\left|\left(\operatorname{Maj}^{[t]} \tilde{i}\right)_{j}-\widetilde{i}_{S}\right| \geq\left|\widetilde{i}_{j}-\widetilde{i}_{S}\right|>0$ and $\widetilde{i}_{j} \neq \widetilde{i}_{S}$. This means that $\left(\operatorname{Maj}^{[t]} \widetilde{i}_{j} \neq \widetilde{i}_{S}\right.$. But $\widetilde{i}_{S}=a$ for certain $a \in A \backslash A^{0}$, and $\left|\widetilde{i^{a}}\right| \geq s \geq t$. Hence, (Maj $\left.{ }^{[t]} \widetilde{i}\right)_{j}=a=\widetilde{i}_{S}$, a contradiction.

Let $S \subseteq N$ be such that $n-t<s<t$, and therefore $s<t$ and $n-s<t$. Hence, for each $i \in I_{S}$, either there exists $a \in A \backslash A^{0}$ such that $\left|i^{a}\right| \geq t$ and $i_{S}=a$, and hence $\left(\mathrm{Maj}^{[t]} i\right)_{j}=a=i_{S}$ for each $j \in N$, or $\left(\mathrm{Maj}^{[t]} i\right)_{j}=i_{j}$ for each $j \in N$.

Note that $S \subseteq F_{\mathrm{Maj}^{[t]}}(S)$, because if $j \in S$, then for each $i \in I_{S}$, either $\left(\mathrm{Maj}^{[t]} i\right)_{j}=i_{S}$ or $\left(\mathrm{Maj}^{[t]} i\right)_{j}=i_{j}=i_{S}$.

Suppose $F_{\mathrm{Maj}}[t](S) \nsubseteq S$. Hence, there is $k \notin S$ such that $k \in F_{\mathrm{Maj}^{[t]}}(S)$, and therefore for each $i \in I_{S},\left(\operatorname{Maj}^{[t]} i\right)_{\underline{i_{j}}}=i_{S}$ if $i_{k}=i_{S}$, and $\left|\left(\mathrm{Maj}^{[t]} i\right)_{k}-i_{S}\right|<\left|i_{k}-i_{S}\right|$ if ${\underset{i}{i_{k}}}_{S} \neq i_{S}$. Take $\tilde{i} \in I_{S}$ such that $\widetilde{i}_{S} \neq \widetilde{i}_{k}=\widetilde{i}_{j}$ for each $j \notin S$. Then we have $\left(\text { Maj }{ }^{[t]} \tilde{i}\right)_{k}=\widetilde{i}_{k} \neq \widetilde{i}_{S}$, a contradiction.

Let $S \subseteq N$ be such that $s \leq n-t$. Suppose that $F_{\mathrm{Maj}^{[t]}}(S) \neq \emptyset$, and let $\widetilde{j} \in F_{\mathrm{Maj}^{j}[t]}(S)$. Hence, for each $i \in I_{S}$, $\left(\mathrm{Maj}^{[t]} i\right)_{\tilde{j}}=i_{S}$ if $i_{\widetilde{j}}=i_{S}$, and $\left|\left(\mathrm{Maj}^{[t]} i\right)_{\tilde{j}}-i_{S}\right|<\left|i_{\tilde{j}}-i_{S}\right|$ if $i_{\widetilde{j}} \neq i_{S}$. Take $\widetilde{i} \in I_{S}$ such that $\widetilde{i}_{S} \neq \widetilde{i}_{k}=a$ for each $k \notin S$ and a certain $a \in A \backslash A^{0}$. Hence, $\widetilde{i^{a}} \mid=n-s \geq t$, and therefore for each $j \in N,\left(\mathrm{Maj}^{[t]} \widetilde{i}\right)_{j}=a \neq \widetilde{i}_{S}$, a contradiction.

(iii) By virtue of (27), we have the following. If $|S| \leq n-t$, then $F_{\mathrm{Maj}[t]}(S)=\emptyset$, and hence $S \notin \mathcal{K}\left(\mathrm{Maj}^{[t]}\right)$. If $|S|=n-t+1$, then $F_{\mathrm{Maj}^{[t]}}(S)=S$, but for each $S^{\prime} \subset S,\left|S^{\prime}\right| \leq n-t$, and therefore $F_{\mathrm{Maj}}\left[S^{\prime}\left(S^{\prime}\right)=\emptyset\right.$. Hence, $S \in \mathcal{K}\left(\mathrm{Maj}^{[t]}\right)$. If $|S|>n-t+1$, then $F_{\mathrm{Maj}^{[t]}}(S) \neq \emptyset$, and there exists $S^{\prime} \subset S$ such that $\left|S^{\prime}\right| \geq n-t+1$, which means that $F_{\mathrm{Maj}[t]}\left(S^{\prime}\right) \neq \emptyset$. Hence, $S \notin \mathcal{K}\left(\mathrm{Maj}^{[t]}\right)$. 
First of all, let us notice that the majority function Maj ${ }^{[t]}$ defined in the multi-choice model is somewhat different from the majority function introduced in the yes-no model; see [20] (Section 5). In the yes-no model, if the majority of players has the inclination +1 , then all players decide +1 , but if not, then all players decide -1 . In the multi-choice model of influence, according to (23), if there is no action $a$ such that the majority of players have the inclination $a$, then each agent follows his own inclination. Consequently, the results on the majority function in the multi-choice model are slightly different from the ones obtained for the yes-no model. One of the differences concerns the set of followers, which additionally, as already mentioned, is defined differently in [20] and in the present paper. As shown in [20], if the cardinality $s$ of coalition $S$ is at least $t$, then each player is a follower of that coalition, but if the cardinality of the coalition is smaller than $t$, then the set of followers of that coalition is empty. In the multi-choice model, as can be seen in (27), if $s$ is at least $t$, then we get the same result as for the yes-no model: every player is a follower of such a coalition. Nevertheless, if $n-t<s<t$, then all members of the coalition and only them are the followers of that coalition. Only coalitions with the cardinality at most $n-t$ have no followers. Consequently, the kernels in both models dif! fer from each other: while in the yes-no model the kernel consists of all coalitions with the cardinality $t$, in the present model the coalitions with exactly $n-t+1$ members create the kernel. Concerning the influence indices under $\mathrm{Maj}^{[t]}$, in [20] we show that the weighted direct influence index of any coalition with the cardinality at least $t$ on a player outside this coalition is equal to 1 . In the present paper we get the similar result: the weighted positive influence index of a coalition with at least $t$ members on a player outside the coalition is also equal to 1 .

Proposition 4 Let $\widetilde{k} \in N$ and consider the guru influence function Gur ${ }^{[\widetilde{k}]}$. Then the following holds:

(i) For each $\emptyset \neq S \subseteq N$ such that $\widetilde{k} \in S$, and for each $j \in N \backslash S$, $D_{\alpha}\left(\operatorname{Gur}^{[\widetilde{k}]}, S \rightarrow j\right)=1$, and $D_{\alpha}^{n e g}\left(\operatorname{Gur}^{[\widetilde{k}]}, S \rightarrow j\right)=0$.

(ii) For each $S \subseteq N$,

$$
F_{\text {Gur }[\tilde{k}]}(S)= \begin{cases}N, & \text { if } \widetilde{k} \in S \\ \emptyset, & \text { if } \widetilde{k} \notin S .\end{cases}
$$

(iii) The kernel is $\mathcal{K}\left(\operatorname{Gur}^{[\widetilde{k}]}\right)=\{\widetilde{k}\}$.

(iv) $\operatorname{Gur}^{[\widetilde{k}]}$ is the unique purely influential function of $\widetilde{k}$ upon $N \backslash \widetilde{k}$, i.e., $\mathcal{B}_{\widetilde{k} \rightarrow N \backslash \widetilde{k}}=\left\{\operatorname{Gur}^{[\widetilde{k}]}\right\}$.

Proof: (iv) Gur ${ }^{[\widetilde{k}]} \in \mathcal{B}_{\widetilde{k} \rightarrow N \backslash \widetilde{k}}$ comes immediately from (19) and (24). Now, $\mathcal{B}_{\widetilde{k} \rightarrow N \backslash \widetilde{k}}$ is reduced to a singleton since $I \backslash I_{\widetilde{k}}=\emptyset$.

(i) Let $\emptyset \neq S \subseteq N$ be such that $\widetilde{k} \in S$, and $j \in N \backslash S$. Hence, in particular for each $i \in I_{S},\left(\operatorname{Gur}^{[\widetilde{k}]} i\right)_{j}=i_{\widetilde{k}}=i_{S}$, which gives $D_{\alpha}\left(\operatorname{Gur}^{[\widetilde{k}]}, S \rightarrow j\right)=1$. Moreover, $\widetilde{I}_{S \rightarrow j}^{\text {neg }}(B)=\emptyset$, ad therefore $D_{\alpha}^{n e g}\left(\operatorname{Gur}^{[\widetilde{k}]}, S \rightarrow j\right)=0$.

(ii) Let $S \subseteq N$ be such that $\widetilde{k} \in S$. Since Gur ${ }^{[\tilde{k}]}$ is purely influential by (iv), $F_{\text {Gur }}^{[\tilde{k}]}$ is an isotone function, and from Prop. 2 (i), we get $F_{\text {Gur }{ }^{[\tilde{k}]}}(S)=N$. 
Let $S \subseteq N$ be such that $\widetilde{k} \notin S$. Suppose that $F_{\text {Gur } \tilde{k}]}(S) \neq \emptyset$. Let $\widetilde{j} \in F_{\text {Gur }}[\tilde{k}](S)$. Hence, for each $i \in I_{S},\left(\operatorname{Gur}^{[\widetilde{k}]}\right)_{\tilde{j}}=i_{S}$ if $i_{\tilde{j}}=i_{S}$. Take $\widetilde{i} \in I_{S}$ such that $\widetilde{i}_{S} \neq \widetilde{i}_{\widetilde{k}}$ and $i_{\tilde{j}}=i_{S}$. Hence, for each $j \in N,\left(\operatorname{Gur}^{[\widetilde{k}} \bar{i}\right)_{j}=\widetilde{i}_{\widetilde{k}} \neq \widetilde{i}_{S}$, and in particular, $\left(\operatorname{Gur}^{[\widetilde{k}} \vec{i}\right)_{\tilde{j}}=\widetilde{i}_{\widetilde{k}} \neq \widetilde{i}_{S}$, a contradiction. (iii) clear from (28).

Since the guru function is defined in the same way both in the yes-no and multichoice models, the results on the set of followers, the kernels, and the influence indices of a coalition with the guru on an 'outside' player are the same in both frameworks.

Proposition 5 Let us consider the identity function Id. Then the following holds:

(i) For each $\emptyset \neq S \subseteq N$ and $j \in N \backslash S, D_{\alpha}(\mathrm{Id}, S \rightarrow j)=D_{\alpha}^{\text {neg }}(\mathrm{Id}, S \rightarrow j)=0$.

(ii) For each $S \subseteq N, F_{\text {ld }}(S)=S$.

(iii) The kernel is $\mathcal{K}(\mathrm{Id})=\{\{k\}, k \in N\}$.

Proof: (i) For each $\emptyset \neq S \subseteq N$ and $j \in N \backslash S,(B i)_{j}=i_{j}$ for $i \in I$, and therefore $D_{\alpha}(\operatorname{ld}, S \rightarrow j)=0$, and $D_{\alpha}^{\text {neg }}(\mathrm{Id}, S \rightarrow j)=0$.

(ii) Note that $S \subseteq F_{\text {Id }}(S)$, because if $j \in S$, then in particular for each $i \in I_{S},(\operatorname{Id} i)_{j}=$ $i_{j}=i_{S}$. Suppose $F_{\text {Id }}(S) \nsubseteq S$. Hence, there is $k \notin S$ such that $k \in F_{\text {Id }}(S)$, and therefore for each $i \in I_{S},(\operatorname{Id} i)_{k}=i_{S}$ if $i_{k}=i_{S}$, and $\left|(\operatorname{Id} i)_{k}-i_{S}\right|<\left|i_{k}-i_{S}\right|$ if $i_{k} \neq i_{S}$. Take $\tilde{i} \in I_{S}$ such that $\widetilde{i}_{S} \neq \widetilde{i}_{k}$. Then we have $\left|(\operatorname{Id} \widetilde{i})_{k}-\widetilde{i}_{S}\right|<\left|\widetilde{i}_{k}-\widetilde{i}_{S}\right|$, but Id $\widetilde{i}=\widetilde{i}$, a contradiction.

(iii) Clear from (ii).

Similar as for the guru function, since the identity function is defined in the same way in the yes-no and multi-choice models, we obtain the same results.

Proposition 6 Let $t \in(0, n]$ and $a \in A \backslash A^{0}$ be fixed, and consider any influence function $B$ in $\mathcal{B}^{[a, t]}$. Then the following holds:

(i) There exists $B \in \mathcal{B}^{[a, t]}$ such that for each $\emptyset \neq S \subseteq N$ and $j \in N \backslash S$,

$$
D_{\alpha}(B, S \rightarrow j)=D_{\alpha}^{n e g}(B, S \rightarrow j)=0 .
$$

(ii) For each $\emptyset \neq S \subseteq N$ such that $s>n-t, t>1$, and $j \in N \backslash S$, there exists $B \in \mathcal{B}^{[a, t]}$ such that

$$
D_{\alpha}(B, S \rightarrow j)=1
$$

(iii) For each $S \subseteq N$,

$$
F_{B}(S) \subseteq S \text { if } s \leq n-t .
$$

Moreover, there exists $B \in \mathcal{B}^{[a, t]}$ such that for each $S \subseteq N, F_{B}(S)=S$.

Proof: (i) Remark that Id $\in \mathcal{B}^{[a, t]}$ for any $a \in A \backslash A^{0}$ and any $t \in(0, n]$. By virtue of Proposition 5 , (i), we get $D_{\alpha}(\mathrm{Id}, S \rightarrow j)=0$ and $D_{\alpha}^{\text {neg }}(\mathrm{Id}, S \rightarrow j)=0$ for each $\emptyset \neq S \subseteq N$ and $j \in N \backslash S$. 
(ii) Take arbitrary $\emptyset \neq S \subseteq N$ such that $s>n-t, t>1$, and $j \notin S$. Define $B$ as follows:

$$
(B i)_{k}= \begin{cases}i_{S}, & \text { if } i \in I_{S \rightarrow j} \text { and } k=j \\ i_{k}, & \text { otherwise }\end{cases}
$$

Note that $B$ belongs to $\mathcal{B}^{[a, t]}$, because if $i \in I_{S \rightarrow j}^{a^{\prime}}$ for $a^{\prime} \neq a$, where

$$
I_{S \rightarrow j}^{a^{\prime}}=\left\{i \in I_{S \rightarrow j} \mid i_{S}=a^{\prime}\right\}
$$

then $\left|i^{a}\right|<t$, and if $i \in I \backslash I_{S \rightarrow j}^{a^{\prime}}$, then (26) is satisfied. We have $D_{\alpha}(B, S \rightarrow j)=1$.

(iii) Let $t \in(0, n], a \in A \backslash A^{0}$, and $s \leq n-t$. Suppose there is $B \in \mathcal{B}^{[a, t]}$ such that $F_{B}(S) \nsubseteq S$ for a certain $S \subseteq N$. This means that $F_{B}(S) \neq \emptyset$, since $\emptyset \subseteq S$ for each $S$. Hence, there is $k \notin S$ such that $k \in F_{B}(S)$. This means that for each $i \in I_{S},(B i)_{k}=i_{S}$ if $i_{k}=i_{S}$, and $\left|(B i)_{k}-i_{S}\right|<\left|i_{k}-i_{S}\right|$ if $i_{k} \neq i_{S}$. Take $\widetilde{i} \in I_{S}$ such that $\widetilde{i}_{S} \neq a, \widetilde{i}_{k}=a$, and $\left|\widetilde{i^{a}}\right| \geq t$. Such an $\widetilde{i}$ always exists, because $n-s \geq t$. Since $\widetilde{\sim}_{\widetilde{i}}^{a} \mid \geq t$, we have $\left.\widetilde{(i}\right)^{a} \subseteq(\widetilde{B i})^{a}$, and therefore $(\widetilde{B i})_{k}=a$, but then $\left|a-\widetilde{i}_{S}\right|=\left|(B \widetilde{i})_{k}-\widetilde{i}_{S}\right|<\left|\widetilde{i}_{k}-\widetilde{i}_{S}\right|=\left|a-\widetilde{i}_{S}\right|$, a contradiction.

If we take $B=\mathrm{Id}$, then for each $S \subseteq N, F_{\mathrm{ld}}(S)=S$.

The mass psychology function in the multi-choice model of influence is a natural generalization of such a function in the yes-no model. Also in the case of this influence function, all results obtained above in Proposition 6 are the same as the ones concerning the yes-no model.

\section{Conclusions}

The main improvement of this paper concerns the enlargement of the set of possible actions in the original framework of influence in a social network, in which players have only two inclinations and two possible decisions. While in the original framework only a yes-no decision was considered, in the present paper we analyze a situation where each player has a totally ordered set of possible actions. A player has an inclination to choose one of the actions, but due to the influence by the others, his decision may be different from his inclination. The main aim of this work, which at the same time emphasizes the innovative elements of the paper, is therefore to generalize the key concepts of the yes-no model of influence (see, [20]) to the framework with an ordered set of possible actions. Consequently, we define the generalized (weighted) influence indices that measure two kinds of influence, i.e., the positive influence and the negative influence. While the positive influence defined in this generalized framework is related to the direct influence studied in the yes-no model, the negative influence is related to the opposite influence defined in $[20]$.

Another important concept of the influence is the concept of follower. While in the original model (see, [20]) the follower of a coalition always decides according to the inclination of that coalition, in the multi-choice model a player is said to be the follower of a coalition if his decision is never farther from the inclination of the coalition. A particular case of the follower that we look at is the perfect follower of a coalition, i.e., a player whose 
decision is always equal to the inclination of the coalition. We check which properties of the set of followers introduced in the yes-no model remain valid in the multi-choice model of influence.

Furthermore, we study another key concept of influence: the influence function. For several influence functions analyzed in [20], we define and study the analogous functions defined in the multi-choice model. The influence functions that we analyze are the majority function, the guru function, the identity function, and the mass psychology function. The results on properties of the majority function defined in the multi-choice framework differ from the ones concerning the original yes-no model, but this is not surprising, since definitions of the majority function for these both models are different. All the remaining influence functions that we consider in this paper have their properties analogous to the properties of the corresponding influence functions in the yes-no framework.

Combining multi-choice problems with influence aspects creates a challenging framework to study. To the best of our knowledge, the concept of the influence indices in the multi-choice model has not been proposed before. Hence, this is an interesting concept from a theoretical point of view, but also its applicability is worth remarking, since in real-life situations frequently a choice of one action from among several (or many) possible actions is to be made.

\section{References}

1. M. J. Albizuri and J. M. Zarzuelo. Coalitional values for cooperative games with $r$ alternatives. TOP, 8:1-30, 2000 .

2. R. Amer, F. Carreras, and A. Magaña. The Banzhaf-Coleman index for games with $r$ alternatives. Optimization, 44:175-198, 1998.

3. E. M. Bolger. Power indices for multicandidate voting games. International Journal of Game Theory, 14:175-186, 1986.

4. E. M. Bolger. A value for games with $n$ players and $r$ alternatives. International Journal of Game Theory, 22:319-334, 1993.

5. E. M. Bolger. A consistent value for games with $n$ players and $r$ alternatives. International Journal of Game Theory, 29:93-99, 2000.

6. E. M. Bolger. Characterizations of two power indices for voting games with $r$ alternatives. Social Choice and Welfare, 19:709-721, 2002.

7. M. Braham and F. Steffen. Voting power in games with abstentions. In M.J. Holler, H. Kliemt, D. Schmidtchen, and M.E. Streit, editors, Power and Fairness, pages 333-348. Mohr-Siebeck, 2002.

8. R. Van den Brink, A. Rusinowska, and F. Steffen. Measures for bipartite directed graphs using success and power in binary voting situations. Mimeo, 2007.

9. R. Van den Brink, A. Rusinowska, and F. Steffen. Measuring power and satisfaction in societies with opinion leaders: Properties of the qualified majority case. Submitted for publication, 2009.

10. P. Dubey and L. S. Shapley. Mathematical properties of the Banzhaf power index. Mathematics of Operations Research, 4:99-131, 1979.

11. D. Felsenthal and M. Machover. Ternary voting games. International Journal of Game Theory, 26:335-351, 1997.

12. D. Felsenthal and M. Machover. The Measurement of Voting Power: Theory and Practice, Problems and Paradoxes. London: Edward Elgar Publishers, 1998.

13. D. Felsenthal and M. Machover. Models and reality: the curious case of the absent abstention. In M. J. Holler and G. Owen, editors, Power Indices and Coalition Formation, pages 87-103. Kluwer, Dordrecht, 2001.

14. P. C. Fishburn. The Theory of Social Choice. Princeton University Press, Princeton, 1973.

15. J. Freixas. Banzhaf measures for games with several levels of approval in the input and output. Annals of Operations Research, 137:45-66, 2005.

16. J. Freixas. The Shapley-Shubik power index for games with several levels of approval in the input and output. Decision Support Systems, 39:185-195, 2005.

17. J. Freixas and W. S. Zwicker. Weighted voting, abstention, and multiple levels of approval. Social Choice and Welfare, 21:399-431, 2003. 
18. M. Grabisch and A. Rusinowska. Influence functions, followers and command games. Submitted for publication, 2008.

19. M. Grabisch and A. Rusinowska. Measuring influence in command games. Social Choice and Welfare, 2009. Forthcoming.

20. M. Grabisch and A. Rusinowska. A model of influence in a social network. Theory and Decision, 2009. Forthcoming.

21. C. Hoede and R. Bakker. A theory of decisional power. Journal of Mathematical Sociology, 8:309-322, 1982.

22. C. R. Hsiao and T. E. S. Raghavan. Shapley value for multichoice cooperative games. Games and Economic Behavior, 5:240-256, 1993.

23. X. Hu and L. S. Shapley. On authority distributions in organizations: controls. Games and Economic Behavior, 45:153-170, 2003.

24. X. Hu and L. S. Shapley. On authority distributions in organizations: equilibrium. Games and Economic Behavior, 45:132-152, 2003.

25. J. R. Isbell. A class of simple games. Duke Mathematical Journal, 25:423-439, 1958.

26. A. Rusinowska. On the not-preference-based Hoede-Bakker index. In L. Petrosjan and V. Mazalov, editors, Game Theory and Applications, volume 13, New York, 2008. Nova Science Publishers, Inc. Forthcoming.

27. A. Rusinowska. The Hoede-Bakker index modified to the Shapley-Shubik and Holler-Packel indices. Group Decision and Negotiation, 2009. Forthcoming.

28. A. Rusinowska and H. De Swart. Generalizing and modifying the Hoede-Bakker index. In H. De Swart, E. Orlowska, G. Schmidt, and M. Roubens, editors, Theory and Applications of Relational Structures as Knowledge Instruments II, LNAI 4342, pages 60-88, Heidelberg, 2006. Springer Verlag.

29. A. Rusinowska and H. De Swart. On some properties of the Hoede-Bakker index. Journal of Mathematical Sociology, 31:267-293, 2007.

30. L. S. Shapley. A Boolean model of organization authority based on the theory of simple games. Mimeo, 1994.

31. B. Tchantcho, L. Diffo Lambo, R. Pongou, and B. Mbama Engoulou. Voters' power in voting games with abstention: Influence relation and ordinal equivalence of power theories. Games and Economic Behavior, 64:335-350, 2008 . 